\title{
Effect of Continuous Quality Care on HAMA, HAMD Score and Quality of Life in Patients with Acute Myocardial Infarction
}

\author{
Yuanle Xu, Hong Wang, Jing Zhang, $\mathrm{Xu} \mathrm{Xu}^{*}$ \\ The Second Affiliated Hospital of Xi'an Medical University, Xi'an, China \\ *Corresponding author: Xu Xu, 1114599241@qq.com
}

Copyright: (C) 2022 Author(s). This is an open-access article distributed under the terms of the Creative Commons Attribution License (CC BY 4.0), permitting distribution and reproduction in any medium, provided the original work is cited.

\begin{abstract}
Objective: To analyze the impact of continuous quality care on HAMA, HAMD score, and quality of life in patients with acute myocardial infarction. Methods: The 100 patients with acute myocardial infarction admitted to our hospital from January 2020 to January 2021 were selected and divided into control and observation groups according to the random number table method, with 50 patients in each group. Patients in the control group were given routine care after discharge, and patients in the observation group received continuous quality care intervention to compare the changes in HAMA, HAMD and quality of life scores before and after care between the two groups. Results: Lower HAMA, HAMD scores and higher quality of life scores compared to care in the observation group, and the difference between groups before and after care was significant $(\mathrm{P}<0.05)$. Conclusion: Continuous quality care can effectively reduce anxiety and depression in AMI patients and improve quality of life, which deserves clinical application.
\end{abstract}

Keywords: Continuous quality care; Acute myocardial infarction; Psychological condition; Quality of life

Online publication: January 19, 2022

\section{Introduction}

Acute myocardial infarction (AMI) is a common coronary syndrome. At present, the most effective clinical treatment of the disease is percutaneous coronary intervention (PCI), and most patients can have more complications and negative emotions such as anxiety and depression after PCI surgery ${ }^{[1]}$. Therefore, it is particularly important to strengthen the care of patients with AMI after PCI. Continuation quality nursing is a kind of off-hospital nursing method that has emerged in recent years. Using information tools and interacting with patients and their families can have unique advantages in promoting patients' physical and mental health ${ }^{[2]}$. This study achieved good continuation care intervention for patients with AMI after discharge, and the results are reported as follows.

\section{Data and methods}

\subsection{General information}

A total of 100 AMI patients admitted to our hospital from January 2020 to January 2021 were selected and divided into control and observation groups according to random number table methods, with 50 patients in each group. This study was carried out after the approval of the hospital ethics committee. There were no significant differences in sex, age, culture, type and site of myocardial infarction $(\mathrm{P}>0.05)$, which were comparable. See Table 1. 
Table 1. General data of the patients in both groups

\begin{tabular}{|c|c|c|c|c|c|c|c|c|c|c|c|}
\hline \multirow[t]{2}{*}{ Group } & \multirow[t]{2}{*}{$\begin{array}{c}\text { Example } \\
\text { number }\end{array}$} & \multirow[t]{2}{*}{$\begin{array}{c}\text { Sex } \\
\text { (male / } \\
\text { female) }\end{array}$} & \multirow[t]{2}{*}{ Age (age) } & \multicolumn{2}{|c|}{$\begin{array}{l}\text { Degree of } \\
\text { education }\end{array}$} & \multicolumn{2}{|c|}{$\begin{array}{c}\text { Type of } \\
\text { myocardial } \\
\text { infarction }\end{array}$} & \multicolumn{4}{|c|}{ Terrier site } \\
\hline & & & & $\begin{array}{c}\text { Primary } \\
\text { school }\end{array}$ & $\begin{array}{l}\text { Junior } \\
\text { middle } \\
\text { school }\end{array}$ & $\begin{array}{c}\text { High } \\
\text { school } \\
\text { or } \\
\text { above }\end{array}$ & $\begin{array}{c}\text { ST } \\
\text { segment } \\
\text { elevation } \\
\text { type }\end{array}$ & $\begin{array}{c}\text { Non-ST } \\
\text { segment } \\
\text { elevation } \\
\text { type }\end{array}$ & Antetheca & $\begin{array}{c}\text { Paries } \\
\text { inferior }\end{array}$ & Other \\
\hline Control group & 48 & $28 / 20$ & $63.82 \pm 4.63$ & 7 & 16 & 25 & 28 & 20 & 19 & 17 & 12 \\
\hline $\begin{array}{l}\text { Observation } \\
\text { group }\end{array}$ & 50 & $31 / 19$ & $64.35 \pm 4.84$ & 6 & 16 & 28 & 32 & 18 & 21 & 18 & 11 \\
\hline
\end{tabular}

\subsection{Inclusion and exclusion criteria}

\subsubsection{Inclusion standards}

(1) Met newly defined global AMI diagnostic criteria in 2013.

(2) Were the first PCI.

(3) No serious complications occurrence.

(4) Voluntarily participated in it and signed the informed consent form.

\subsubsection{Exclusion criteria}

(1) Has malignant heart arrhythmias, heart valve disease and other heart diseases.

(2) Combined with the heart, brain, liver, kidney and other important organ dysfunction.

(3) Combined with hypertension, malignant tumor, recent infection, chronic obstructive pulmonary disease.

(4) Mentally disturbed.

(5) Contraindication to PCI.

\subsection{Method}

Routine care: Regular review at the 1st, 3rd, 6th and 12th months after discharge. Implementation of continuing care intervention:

\subsubsection{Regular telephone return visit}

Return visits, once a month per week, 1 month after discharge, 2 to 3 months after discharge, 1 half month, 1 monthly visit at 15-30 min for a total of 1 year. The contents of the return visit include understanding the patient's condition, medical drug compliance, exercise, recent cardiovascular event recurrence and revisit, Explain the knowledge related to the disease, Ask the patient to insist on medication and talk about its importance, Explain the administration method and clinical significance of PCI related drugs (such as statin lipid drugs, antiplatelet drugs, etc.). For irregular patient behavior, give timely guidance, and according to the patient completed the previous health target to give the patient timely affirmation and encouragement. Focus on the patient's psychological state, and give targeted psychological support according to the situation, Including relaxation training, music therapy, and behavioral training. Targeted guidance of patient's diet and defecation. Patient families were encouraged to actively participate in this procedure during the above intervention, and he was trained in CPR, In response to patient emergencies.

\subsubsection{Regular home visits}

Ask again for the above content, and emphasize that the frequency of home visits is consistent with the 
telephone return visit, but the two are interspersed.

\subsubsection{Come to the hospital for reexamination}

Within half a year after discharge, have a second visit every 1 to 2 months, half a year after discharge, every six months. If the abnormal condition is abnormal, see a doctor immediately.

\subsection{Observing indicators}

Anxiety and depression were assessed using the Hamilton Anxiety Scale (Hamilton anxiety scale, HAMA) ${ }^{[3]}$, Hamilton Depression Scale (Hamilton Depression Scale, HAMD) ${ }^{[4]}$, The higher the score, the worse the negative mood. QOL evaluation using WHO QO of Life Scale (WHO-QOL-BREF) ${ }^{[5]}$, The higher the total score, it represents a good quality of life.

\subsection{Statistical methods}

$X$ Raw data were stored and processed by SPSS 19.0, mean \pm standard deviation $(X \pm \mathrm{s})$, data differences between groups were analyzed by parallel t-test, percentage (\%), data differences were analyzed by parallel chi-square test, $\mathrm{P}<0.05$.

\section{Result}

\subsection{Comparison of HAMA, HAMD scores before and after care between the two groups}

Before care, HAMA and HAMD scores were insignificant $(\mathrm{P}>0.05)$ in both groups, HAMA and HAMD scores were lower compared with pre-care, and more significant in observation groups, within and between groups $(\mathrm{P}<0.05)$. See Table 2.

Table 2. HAMA, HAMD scores before and after care of both groups (points)

\begin{tabular}{lccccc}
\hline Group & Example & \multicolumn{2}{c}{ HAMA } & \multicolumn{2}{c}{ HAMD } \\
\cline { 2 - 5 } & number & Before nursing & After nursing & Before nursing & After nursing \\
\hline Control group & 48 & $25.45 \pm 5.31$ & $19.89 \pm 3.77$ & $26.67 \pm 5.15$ & $21.95 \pm 3.62$ \\
Observation group & 50 & $25.12 \pm 5.16$ & $16.65 \pm 3.46$ & $26.34 \pm 5.04$ & $17.90 \pm 3.27$ \\
\hline
\end{tabular}

\subsection{Comparison of quality of survival scores before and after care between the two groups}

Before care, the QOL scores ( $\mathrm{P}>0.05)$ were not different, the QOL scores were higher than before care, and more significantly in observation groups, within and between groups $(\mathrm{P}<0.05)$. See Table 3 for details.

Table 3. Quality of survival score before and after care of patients in the two groups (points)

\begin{tabular}{lccc}
\hline Group & Example number & Before nursing & After nursing \\
\hline Control group & 48 & $46.98 \pm 6.10$ & $53.32 \pm 6.76$ \\
Observation group & 50 & $47.23 \pm 5.96$ & $58.47 \pm 7.04$ \\
\hline
\end{tabular}

\section{Discussion}

With China's aging society, as well as the acceleration of modern production and life pace and psychological factors, the incidence of AMI in China has increased year by year in the past 10 years, and has become one of the main causes of death. The implementation of percutaneous coronary intervention in AMI patients can effectively play the role of revascularization, improve myocardial ischemia, and then relieve the condition. However, patients still need to take medicine, diet and exercise, as well as maintain 
a psychological balance, so as to effectively prevent disease recurrence. However, some patients lack good cognitive ability and have a heavy psychological burden. The implementation of the rehabilitation plan after discharge is not standardized, which seriously affects the treatment and rehabilitation effect [6] Therefore, it is necessary to implement a continuous care intervention for patients after AMI intervention after surgery.

Continuation nursing is a kind of nursing mode in recent years, can extend the hospital medical services to the family, is the continuation of the hospital nursing, ensure that patients can still accept correct collaborative nursing intervention, through regular telephone visit, family follow-up for later care, to strengthen the discharge medicine, exercise, diet and complication prevention knowledge, make patients realize the importance of disease treatment, and teach patients and their families necessary first aid skills [7]. The purpose of continuity care is to establish effective self-management through education and effective guidance, take correct medication, visit regular visits and follow doctor's advice, so as to delay the development of disease and improve the quality of life. Clinical studies have confirmed that carrying out quality care in continuous care work can relax the patients' physical and psychological state, which is conducive to early rehabilitation and improve the quality of life ${ }^{[8]}$.

In view of this, this study gave continuous quality care for AMI patients after discharge. The results showed that HAMA and HAMD scores were lower than before treatment, and the quality-of-life score was higher than that of control group, respectively. The difference was statistically significant $(\mathrm{P}<0.05)$, suggesting that continuity quality care can improve the psychological mood and quality of life of AMI patients after discharge, and the nursing effect is ideal than routine care. Tabulation that this may be related to continuous quality care to strengthen the knowledge and ability to strengthen disease self-care of patients and their families, as well as narrowing the care relationship. The report showed that continuous quality care for AMI patients undergoing PCI showed significantly reduced anxiety and significantly improved treatment compliance ${ }^{[9]}$, similar to the results of this study, jointly confirming that continuity quality care can ensure patient quality of life after discharge. The remission of adverse mood is beneficial to reducing its impact on disease treatment and increasing patient confidence in fighting the disease to promote speedy recovery.

\section{Conclusion}

In conclusion, continuous quality care can effectively reduce anxiety and depression, improve improvement of prognosis and improve quality of life in AMI patients.

\section{Funding}

Fund project no.: 21JK0897

\section{Disclosure statement}

The author declares no conflict of interest.

\section{References}

[1] Zhang L, 2017, Observation on the Effect of Quality Care Mode in Improving the Quality of Survival of Patients with Acute Myocardial Infarction. Chinese Medical Guidelines, 15(6): 218.

[2] Wang Y, Liu K, Qu X, 2017, Study of Effect of Continuation Care Intervention in Patients with PCI with Acute Myocardial Infarction. Journal of Practical Clinical Medicine, 21(4): 167-170.

[3] Yue X, 2016, Analysis of the Effect of Continuation Care on Depressive Anxiety in Patients with 
Acute Myocardial Infarction. Chinese Practical Medicine, 11(7): 220-221.

[4] He C, Liang X, Qu Y, 2016, Observation of the Effect of Continuation Care in Patients with Coronary Intervention for Acute Myocardial Infarction. Chinese Medical Guide, 13(1): 159-162.

[5] Xu Z, Zhu Y, Yue S, et al., 2016, Clinical Effectiveness of Quality Care in Alleviating Anxiety in Patients with Acute MI. Chinese Pharmaceutical Guide, 18 (2): 195-196.

[6] Yan H, Wang X, 2021, Clinical Application Value of Continuation Care Mode after Intervention of Acute Myocardial Infarction. Henan Journal of Surgery, 2021,04:176-177.

[7] Wei L, 2016, Application of Continuity Care in the Care of Patients with Acute Myocardial Infarction. Practical Medicine Journal, 15(9): 836-838.

[8] Chen T, Fei Z, Wei W, 2016, Application Value of the Omaha System in the Care of Acute Myocardial Infarction in the Elderly. Practical Geriatrics, 15(7): 612-614.

[9] Jia H, 2016, Effects of Continuation Care on Psychological Status and Prognosis in Elderly Patients with Acute Myocardial Infarction. E-Journal of Clinical Medical Literature, 3(22): 4431-4432.

Publisher's note

Bio-Byword Scientific Publishing remains neutral with regard to jurisdictional claims in published maps and institutional affiliations. 